J. Appl. Numer. Optim. 3 (2021), No. 2, pp. 403-424

Available online at http://jano.biemdas.com

https://doi.org/10.23952/jano.3.2021.2.09

\title{
A DYNAMIC SUPPLY CHAIN NETWORK FOR PPE DURING THE COVID-19 PANDEMIC
}

\author{
P. DANIELE*, D. SCIACCA \\ Department of Mathematics and Computer Science, University of Catania, Catania, Italy
}

\begin{abstract}
In this paper, we present an optimization model consisting of a dynamic supply chain network related to Personal Protective Equipment (PPE). We suppose that the variables in the model, namely flows on arcs and additional capacities on arcs, depend both on time and on a delay function. The aim of the firm is to find the optimal flows and the optimal additional capacities on arcs to satisfy the huge and immediate increasing request in the demand markets due to the spread of the COVID-19 disease, minimizing, simultaneously, its total costs. We obtain a minimization problem and the related "retarded" evolutionary variational inequality (rEVI). We introduce the associated infinite-dimensional projected dynamical system to obtain a computational procedure to find the optimal solution to the rEVI associated with our minimization problem and, finally, we propose some numerical examples based on real scenarios.
\end{abstract}

Keywords. COVID-19; Dynamic supply chain network; Evolutionary variational inequalities; Minimization problem.

\section{INTRODUCTION}

On January 9, 2020, the World Health Organization announced the identification of a new coronavirus never before identified in humans, later dubbed SARS-CoV-2. In a few months, this virus has spread to all continents and the various countries have implemented, following the indications of the World Health Organization, various strategies to mitigate its spread. Commonly used measures was social distancing, total or partial lockdowns, and the use of surgical masks.

The advent of Sars-CoV-2 pandemic has profoundly changed the habits of all citizens, making surgical masks and, more generally, PPE, devices for daily use. The global scope of this event allows us to categorize the Sars-CoV-2 pandemic as a disaster scenario. In terms of disaster preparedness, the procurement of PPE has been an important and difficult to manage problem since the beginning of the pandemic (see, for instance, [1]). It is clear that the demand for these devices has changed over time: if before the epidemic, medical-surgical masks and/or masks for specific use were used only by medical personnel or workers specialized in sectors where the use of protections was necessary, as the pandemic progresses, the request has been

${ }^{*}$ Corresponding author.

E-mail addresses: patrizia.daniele@ unict.it (P. Daniele), daniele.sciacca@unipa.it (D. Sciacca).

Received October 31, 2020; Accepted April 6, 2021.

(C)2021 Journal of Applied and Numerical Optimization 
extended, gradually, to the entire world population. The industries that manufacture and supply such devices had to adapt their production and supply capacities to the huge and sudden increase in request in the demand markets. This adaptation required time and the use of additional resources which led, in most cases, to delays in the supply or to a partial supply of the product. Moreover, the adaptation of manufacturing involves costs to increase the production, distribution and storage of the product and, furthermore, costs to increase the capacity of the arcs that form the network. However, the supply of the product is regulated by the capacity of the arcs that form the network. For this reason, in this paper, we will assume that a PPE manufacturer has the possibility of increasing the capacity of its links by deciding to invest in this increase.

In the existing literature, several optimization models based on supply chains have dealt with the problem of the supply of basic necessities (see, for istance, [2, 3, 4, 5]). More specifically, in [6], an integrated framework for the design of supply chain networks for critical products such as vaccines, medicines, food, etc., which may be used in preparation (and response) to pandemics, disasters, attacks, etc. In [7], Nagurney and Nagurney extended the model of [8] by including stochastic link costs, and with the objective function including the minimization of the expected costs as well as the variance with an associated weight for the latter to denote the humanitarian organization's value of risk reduction. Also, in [9], He and Zhuang constructed a two-stage, dynamic model to assess the trade-off between pre-disaster preparedness and post-disaster relief with the goal of minimizing the total expected damage and the costs of preparedness and relief. Finally, in [10], Nagurney and Salarpour constructed the first stochastic Generalized Nash Equilibrium model for the study of competition among countries for limited supplies of medical items (PPEs, ventilators, etc.) in the disaster preparedness and response phases in the Covid-19 pandemic.

In the aforementioned models, the decision makers' goal was to minimize expected costs or maximize expected profits. In any case, all these models are affected by a degree of uncertainty due to the sudden advent of a disastrous scenario, which forces companies, suppliers, countries and humanitarian organizations to modify their production plan to cope with a huge request increase in the demand markets.

As previously discussed, this adaptation required time and the use of additional resources which led, in most cases, to delays in the supply or to a partial supply of the product. In [11], the delay effects in traffic network models are described. Since the information through the network travels at finite speed, then the users take a certain time before adjusting their path choices and reaching an equilibrium state. In our model, the possible drastic increase in demand at the demand markets could result in a delay in the supply of PPE by the producer. As a consequence, it is reasonable to think that demand requirements at time $t$ are satisfied only in a subsequent time affected by a delay $h$. In general (see, for example, $[12,13,14]$ ) for sake of simplicity, the delay $h$ is assumed to be a positive constant value. In [15], instead, Scrimali considered a delay vector that depends both on time and on length of route.

Our contribution consists in considering a delay vector that depends both on time and on the firm reaction time to the sudden request. In this way, it is possible to describe how the suppliers actually adapt their supply to the real requests coming from the demand markets. Furthermore, we will assume that the request in the demand markets is proportional to the number of infections and, therefore, the suppliers know the real need for such devices in the 
demand markets and act in terms of expanding production and distribution arcs to satisfy such requests.

This paper is organized as follows. In Section 2, we present and study the model in an time interval $[0, T]$, and we introduce the cost functions associated with management and capacity increase of production, distribution and storage arcs of the network. We suppose that arc and path flows are affected by a delay that, in turn, depends both on time and on the firm reaction time to the sudden request in demand markets, whose requests are assumed proportional to number of positive cases. We determine optimality conditions for the firm and derive the evolutionary variational inequality (EVI) formulation associated to our problem. Then we give conditions of existence and uniqueness of the solutions to the EVI. In Section 3, we introduce the associated infinite-dimensional Projected Dynamical System (PDS) and describe the relation between EVI and PDS, which allows us to obtain a computation procedure that we apply in the successive Section 4 to solve numerical examples which describe real scenarios of application of our model. We also conduct both a statistical and a sensitivity analysis on arc flows. Section 5 is dedicated to the conclusions.

\section{Mathematical Model}

In this Section, we describe the dynamic supply chain network model for manufacturing and distribution of Personal Protective Equipment (PPE). The network topology is depicted in Figure 1. We assume that the supply chain network consists of $M$ manufacturing plants, represented by the nodes of the top level; $D$ distribution centers, represented by the nodes of the second level that have to serve the $K$ demand markets, denoted by $Z_{1}, \ldots, Z_{k}, \ldots, Z_{K}$ represented by the nodes of the bottom level. We assume that distribution centers may have $D$ distribution storage centers. The links from the manufacturing nodes, denoted by $X_{1}, \ldots, X_{m}, \ldots, X_{M}$, are connected to the possible distribution center nodes of the firm, denoted by $Y_{1,1}, \ldots, Y_{d, 1}, \ldots, Y_{D, 1}$. These links correspond to the possible shipment links between the manufacturing plants and the distribution centers. The links that connect the nodes $Y_{d, 1}, d=1, \ldots, D$ with the nodes $Y_{d, 2}$, $d=1, \ldots, D$ correspond to the possible storage links. Finally, there are possible shipment links between the nodes $Y_{d, 2}, d=1, \ldots, D$, and the demand nodes $Z_{k}, k=1, \ldots, K$.

We denote by $G=[N, L]$ the supply chain network, where $N$ is the set of nodes and $L$ represents the set of links. We further denote by $n_{L}$ the number of links in the link set $L$. We suppose to partition the set $L$ as follows: $L=L_{1} \cup L_{2}, L_{1} \cap L_{2}=\emptyset$, where $L_{1}$ indicates the set of links which the firm can add capacity to and $L_{2}$ the set of links that have a fixed existing capacity. We denote the links by $a, b, \ldots$ and $\alpha, \beta, \ldots$

Let $w_{k}$ denote a pair of origin/destination (O/D) nodes $\left(1, Z_{k}\right)$ and let $P_{w_{k}}$ denote the set of paths that connect the $\mathrm{O} / \mathrm{D}$ pair $\left(1, Z_{k}\right)$ through the existing nodes, where $k=1, \ldots, K$. We denote by $P$ the set of network paths, where

$$
P=\bigcup_{k=1, \ldots, K} P_{w_{k}}
$$

Let us suppose that the functional space for the trajectories of link flows is $L^{2}\left(I, \mathbb{R}_{+}^{|L|}\right)$ while for the trajectories of the path flows is $L^{2}\left(I, \mathbb{R}_{+}^{|P|}\right)$, where $I \subseteq \mathbb{R}$.

With each path $p \in P$ of the network we associate a flow of product, $F_{p} \in L^{2}\left(I, \mathbb{R}_{+}\right)$, that represents the flow of product on path $p$ joining node 1 with a demand node. We group these 


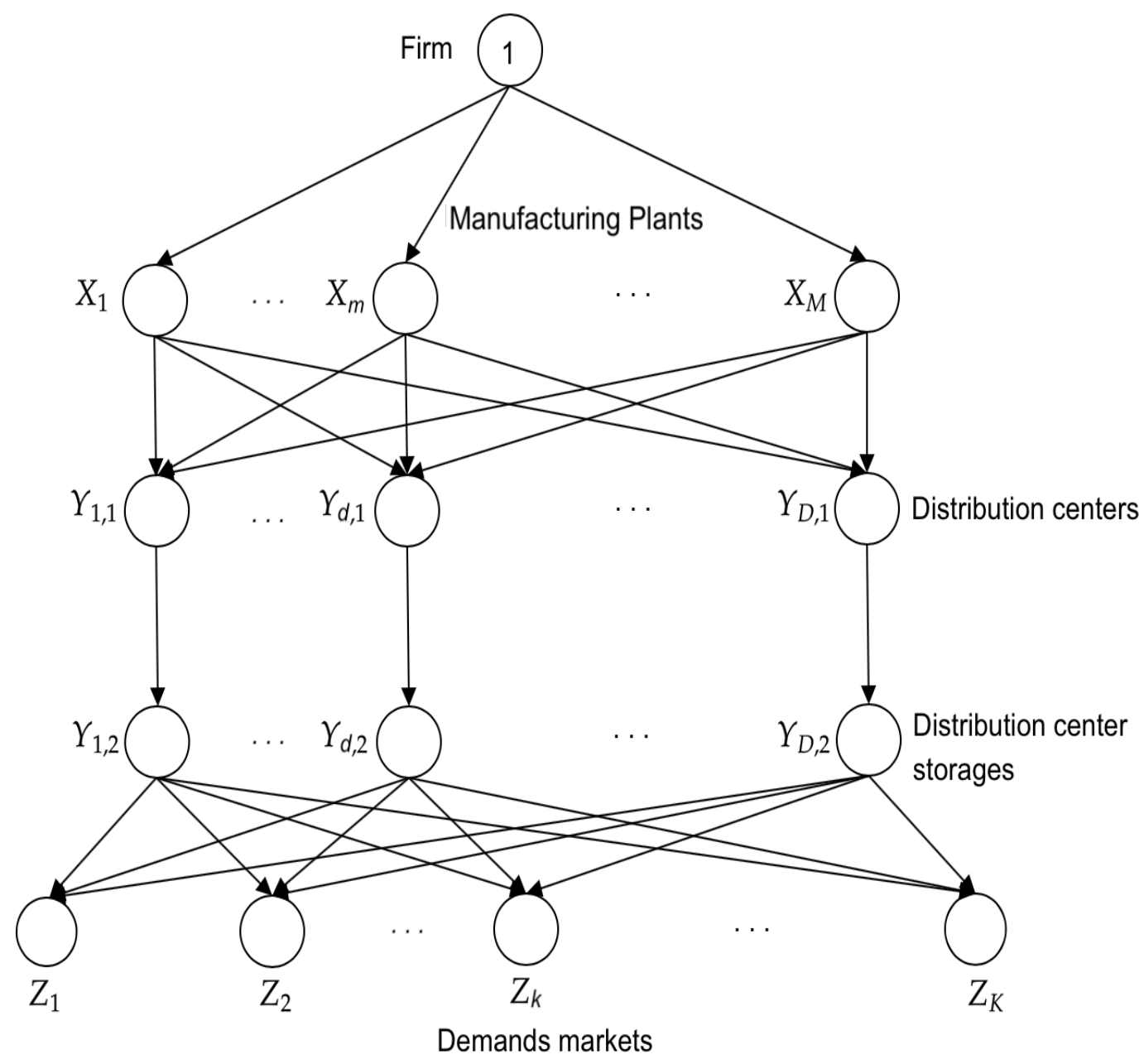

FIGURE 1. Supply Chain Network Topology.

quantities into the vector

$$
F(\cdot)=\left(F_{p}(\cdot)\right)_{p \in P} \in L^{2}\left(I, \mathbb{R}_{+}^{|P|}\right) .
$$

Let $f_{a}(\cdot)$ denote the flow of the product on link $a$. We group these quantities in the vector $f(\cdot)=\left(f_{a}(\cdot)\right)_{a \in L} \in L^{2}\left(I, \mathbb{R}_{+}^{|L|}\right)$. We suppose that a link flow is the sum of path flows that contain that link. Hence, we must satisfy the following conservation of flow equations:

$$
f_{a}(\cdot)=\sum_{p \in P} F_{p}(\cdot) \delta_{a p}, \quad \forall a \in L, \text { a.e. } v \in I,
$$

where $\delta_{a p}$ represents an element of incidence matrix of link $a$ on path $p$. Namely, for every link, the link flow is equal to the sum of the flows on paths which contain such a link. We can rewrite equation (2.2) as follows:

$$
f(\cdot)=\Delta F(\cdot),
$$

where $\Delta$ represents the incidence matrix of link $a$ on path $p$.

We suppose that every link $a \in L$ has an existing capacity of flow, that we indicate with $\bar{u}_{a}(\cdot) \in L^{2}\left(I, \mathbb{R}_{+}\right)$. 
The approach that we utilize in this paper is that of system optimization, where the firm aims at minimizing the total costs associated with its production, storage, distribution activities in order to satisfy, at every time, the request at the demand points. In this paper, we shall suppose that request of every demand market vary over time as a function of the trend of the contagion curve in the same demand market. In such a way, we will describe how the firm adapts its activity in response to a sudden change of request.

As discussed in the Introduction, in this model we suppose that the delay depends both on time and on the firm reaction time to the sudden request.

Let us define the firm reaction time to the sudden request in demand markets, $w$, and we suppose that it is a function of the time, i.e.,

$$
w:=w(t) .
$$

We can now introduce the delay $h$ and we suppose that it is a function of the departure time $t \in[0, T]$ and the firm reaction time:

$$
h:=h(t, w(t)) \in \mathbb{R}_{+}, \quad t \in[0, T] .
$$

In our model, we assume that the delay is nonnegative; thereby we do not consider early arrivals. We also suppose that $h(t, w(t))$ is a nondecreasing linear function with respect to $t$. By doing this we will avoid the unfavorable case in which the delay increases and the network suffers a blockage.

We can introduce the retarded flow on links:

$$
f(\tau)=\left(f_{a}(t+h(t, w(t)))\right)_{a \in L}:[0, T] \rightarrow \mathbb{R}_{+}^{|L|},
$$

and the retarded flow on paths:

$$
F(\tau)=\left(F_{p}(t+h(t, w(t)))\right)_{p \in P}:[0, T] \rightarrow \mathbb{R}_{+}^{|P|} .
$$

Remark 2.1. We want to stress that $h(t, w(t)) \in L^{2}\left([0, T], \mathbb{R}_{+}\right)$as it is a nonnegative, nondecreasing with respect to time and bounded function and hence it is measurable and Lebesgueintegrable. Therefore, since $f(t+h(t, w(t)))$ and $F(t+h(t, w(t)))$ are in turn nonnegative, non decreasing with respect to time and bounded, it results that $f(t+h(t, w(t))) \in L^{2}\left([0, T], \mathbb{R}_{+}^{|L|}\right)$ and $F(t+h(t, w(t))) \in L^{2}\left([0, T], \mathbb{R}_{+}^{|P|}\right)$.

For simplicity of notation we put $\tau=t+h(t, w(t))$. Hence, we can rewrite the conservation law (2.2) as follows:

$$
f_{a}(\tau)=\sum_{p \in P} F_{p}(\tau) \delta_{a p}, \quad \forall a \in L, \text { a.e. } t \in[0, T]
$$

Let $d_{k}(t) \in L^{2}\left([0, T], \mathbb{R}_{+}\right)$denote the demand at demand market $Z_{k}, k=1, \ldots, K$, at time $t \in[0, T]$ and we group these quantities into the vector

$$
d(t)=\left(d_{k}(t)\right)_{k=1, \ldots, K} \in L^{2}\left([0, T], \mathbb{R}_{+}^{K}\right) .
$$

As already announced, we suppose that the demands in the demand markets at every time $t \in[0, T]$ are satisfied with a delay factor.

Therefore, we suppose that the following constraint is satisfied:

$$
\sum_{p \in P_{w_{k}}} \delta_{a p} F_{p}(\tau) \geq d_{k}(t), \quad k=1, \ldots, K \text {, a.e. } t \in[0, T]
$$


which means that for every O/D pair $w_{k}$ the sum of the flows on all paths connecting $w_{k}$ at the delayed time $\tau$ is no less than the demand at time $t$.

We make the following fundamental assumption:

Assumption 2.1. We suppose that demand at every demand market and at every time $t \in[0, T]$ is a function of the trend of infections in the areas served by the same demand market.

Therefore, we assume that

$$
d_{k}(t)=l_{k} I_{k}(t), \quad \forall k=1, \ldots, K \text { and a.e. } t \in[0, T],
$$

where $l_{k} \in[1,10] \cap \mathbb{N}, k=1, \ldots, K$, represents a proportionality coefficient which expresses the relationship between population, number of swabs performed and positive cases found and $I_{k}(t) \in L^{2}\left([0, T], \mathbb{R}_{+}\right)$is the function representing the trend of infections that we suppose to be a convex and differentiable function. This assumption is justified by the concept of diminishing marginal utility, according to which, roughly speaking, averages are better than the extremes.

According to equation (2.5), we can rewrite constraint (2.4) as follows:

$$
\sum_{p \in P_{w_{k}}} \delta_{a p} F_{p}(\tau) \geq l_{k} I_{k}(t), \quad k=1, \ldots, K, \text { a.e. } t \in[0, T] .
$$

Let $c_{a}(t)$ be the total cost on a link at time $t \in[0, T]$, be it a production link, a shipment link or a storage link and we assume $c_{a}(t)$ as a function of $f_{a}(t)$ :

$$
c_{a}(t)=c_{a}\left(f_{a}(t)\right), \quad \forall a \in L, \text { a.e. } t \in[0, T] .
$$

We introduce the existing retarded capacity $\bar{u}_{a}(\tau)$ and we observe that the vector

$$
\bar{u}(\tau)=\left(\bar{u}_{a}(\tau)\right)_{a \in L} \in L^{2}\left([0, T], \mathbb{R}_{+}^{|L|}\right) .
$$

For every link $\alpha \in L_{1}$, we denote by $u_{\alpha}(t) \in L^{2}\left([0, T], \mathbb{R}_{+}\right)$the added capacity by the firm in this link and we group these quantities into the vector

$$
u(t)=\left(u_{\alpha}(t)\right)_{\alpha \in L_{1}} \in L^{2}\left([0, T], \mathbb{R}_{+}^{\left|L_{1}\right|}\right) .
$$

We suppose that additional capacities are added, that is:

$$
u(t) \leq \lambda(t), \text { a.e. } t \in[0, T],
$$

where $\lambda(t)=\left(\lambda_{\alpha}(t)\right)_{\alpha \in L_{1}}$.

Let $\pi_{\alpha}(t)$, for every $\alpha \in L_{1}$ and a.e. $t \in[0, T]$, be the investment cost of adding capacity on a link $\alpha$ and assume that it is a function of the added capacity on the link, i.e.

$$
\pi_{\alpha}(t)=\pi_{\alpha}\left(u_{\alpha}(t)\right), \quad \forall \alpha \in L_{1}, \text { a.e. in }[0, T] .
$$

In this model our aim is to minimize the total costs incurred by the firm to adapt in an efficient way the product flows and capacity of links to a spread of demand of PPE in the demand market related to the outbreak of COVID19 epidemic. So the optimization problem reads as follows:

$$
\min \left\{\sum_{a \in L} c_{a}\left(f_{a}(\tau)\right)+\sum_{\alpha \in L_{1}} \pi_{\alpha}\left(u_{\alpha}(t)\right)\right\}
$$


subject to constraints:

$$
\begin{gathered}
f_{a}(\tau)=\sum_{p \in P} F_{p}(\tau) \delta_{a p}, \quad \forall a \in L, \text { a.e. } t \in[0, T] \\
f_{\alpha}(\tau) \leq \bar{u}_{\alpha}(\tau)+u_{\alpha}(t), \quad \forall \alpha \in L_{1}, \text { a.e. } t \in[0, T] \\
u_{\alpha}(t) \leq \lambda_{\alpha}(t), \quad \forall \alpha \in L_{1}, \text { a.e. } t \in[0, T] \\
f_{\beta}(\tau) \leq \bar{u}_{\beta}(\tau), \quad \forall \beta \in L_{2}, \text { a.e. } t \in[0, T] \\
\sum_{p \in P_{w_{k}}} \delta_{a p} F_{p}(\tau) \geq l_{k} I_{k}(t), \quad k=1, \ldots, K, \text { a.e. } t \in[0, T] .
\end{gathered}
$$

We denote by $\gamma(x(t))$ the objective function:

$$
\gamma(x(t))=\sum_{a \in L} c_{a}\left(f_{a}(\tau)\right)+\sum_{\alpha \in L_{1}} \pi_{\alpha}\left(u_{\alpha}(t)\right)
$$

and consider the feasible vectors

$$
x(t)=(f(\tau), u(t)) \in \mathscr{L}=L^{2}\left([0, T], \mathbb{R}_{+}^{|L|}\right) \times L^{2}\left([0, T], \mathbb{R}_{+}^{\left|L_{1}\right|}\right)
$$

and the feasible set

$$
\begin{aligned}
& \mathbb{K}:=\left\{x(t) \in \mathscr{L}: \exists F(\tau) \in L^{2}\left([0, T], \mathbb{R}_{+}^{|P|}\right)\right. \text { and } \\
& f_{a}(\tau)=\sum_{p \in P} F_{p}(\tau) \delta_{a p}, \quad \forall a \in L, \text { a.e. } t \in[0, T] \\
& f_{\alpha}(\tau) \leq \bar{u}_{\alpha}(\tau)+u_{\alpha}(t), \quad \forall \alpha \in L_{1} \text {, a.e. } t \in[0, T] \\
& u_{\alpha}(t) \leq \lambda_{\alpha}(t), \quad \forall \alpha \in L_{1} \text {, a.e. } t \in[0, T] \\
& f_{\beta}(\tau) \leq \bar{u}_{\beta}(\tau), \quad \forall \beta \in L_{2} \text {, a.e. } t \in[0, T] \\
& \left.\sum_{p \in P_{w_{k}}} \delta_{a p} F_{p}(\tau) \geq l_{k} I_{k}(t), \quad k=1, \ldots, K, \text { a.e. } t \in[0, T]\right\} \text {. }
\end{aligned}
$$

We observe that $\mathbb{K}$ is a convex, closed and bounded set. Moreover, if we require that

$$
\sum_{k \in \mathbb{K}} l_{k} I_{k}(t)<\min \left\{\bar{u}_{a}(\tau)+u_{a}(t), \lambda_{a}(t)\right\}
$$

the set $\mathbb{K}$ is nonempty.

Further, we require that for all $f \in \mathbb{R}_{+}^{|L|}$ and for all $u \in \mathbb{R}_{+}^{\left|L_{1}\right|}$, the following growth conditions hold true:

$$
\left|c_{a}(f)\right| \leq \varepsilon_{a}\|f\| \text { and }\left|\pi_{a}(u)\right| \leq \vartheta_{a}\|u\|
$$

and

$$
\left|\frac{\partial c_{a}(f)}{\partial f_{a}}\right| \leq \mu_{a} \text { and }\left|\frac{\partial \pi_{a}(u)}{\partial u_{a}}\right| \leq \eta_{a}
$$

where $\varepsilon_{a}, \vartheta_{a}, \mu_{a}$ and $\eta_{a}$ are positive real numbers.

The following theorem holds (see also [16]): 
Theorem 2.1. If $c_{a}(\cdot)$ and $\pi_{\alpha}(\cdot)$ are convex and continuously differentiable functions in $f$ and in $u$, for all $a \in L$ and for all $\alpha \in L_{1}$, respectively, and if the growth conditions (2.14) and (2.15) are satisfied, then $x^{*}(t) \in \mathbb{K}$ is a retarded solution to problem (2.6)-(2.11) if and only if it is a solution to the retarded evolutionary variational inequality:

$$
\begin{gathered}
\text { Find } x^{*}(t)=\left(f^{*}(\tau), u^{*}(t)\right) \in \mathbb{K} \text { such that } \\
\int_{0}^{T} \sum_{a \in L}\left[\frac{\partial c_{a}\left(f^{*}(\tau)\right)}{\partial f_{a}(\tau)}\right] \times\left[f_{a}(\tau)-f_{a}^{*}(\tau)\right] d t \\
+\int_{0}^{T} \sum_{\alpha \in L_{1}}\left[\frac{\partial \pi_{\alpha}\left(u_{a}^{*}(t)\right)}{\partial u_{\alpha}(t)}\right] \times\left[u_{\alpha}(t)-u_{\alpha}^{*}(t)\right] d t \geq 0 \\
\forall x(t)=(f(\tau), u(t)) \in \mathbb{K} .
\end{gathered}
$$

Proof. Since $c_{a}(\cdot)$ and $\pi_{\alpha}(\cdot)$ are convex and continuously differentiable for all $a \in L$ and for all $\alpha \in L_{1}$ by assumption, then the function $\gamma(x(t))$ is a convex and continuously differentiable function. The following condition is necessary and sufficient to ensure that $x^{*}(t)$ solves the minimization problem (2.6)-(2.11) (see Theorem 5 in [17]):

$$
\begin{aligned}
& \sum_{a \in L}\left[\frac{\partial c_{a}\left(f^{*}(\tau)\right)}{\partial f_{a}(\tau)}\right] \times\left[f_{a}(\tau)-f_{a}^{*}(\tau)\right] \\
& +\sum_{\alpha \in L_{1}}\left[\frac{\partial \pi_{\alpha}\left(u_{\alpha}^{*}(t)\right)}{\partial u_{\alpha}(t)}\right] \times\left[u_{\alpha}(t)-u_{\alpha}^{*}(t)\right] \geq 0, \quad \forall x(t) \in \mathbb{K}, \text { a.e. in }[0, T] .
\end{aligned}
$$

Therefore, by integrating in the time interval $[0, T]$, we get the time-dependent variational inequality (2.16).

For the proof of vice versa see Theorem 1 in [16].

For what concerns the existence, we may provide some existence theorems under general assumptions. Let us recall the following definitions (see, for example, [11, 18, 19, 20, 21]). Let $E$ be a real topological vector space, and $\mathbb{K} \subseteq E$ convex. Then $C: \mathbb{K} \rightarrow E^{*}$ is said to be:

- pseudomonotone in the sense of Karamardian iff, for all $x, y \in \mathbb{K}$,

$$
\langle C(x), y-x\rangle \geq 0 \Rightarrow\langle C(y), x-y\rangle \leq 0 ;
$$

- hemicontinuous in the sense of Ky-Fan iff, for all $y \in \mathbb{K}$, the function $\xi \mapsto\langle C(\xi), y-\xi\rangle$ is upper semicontinuous on $\mathbb{K}$;

- hemicontinuous along line segments iff, for all $x, y, z \in \mathbb{K}$, the function $t \mapsto\langle C(t x+(1-$ $t) y), z\rangle$ is continuous.

The general theorem related to the existence of the solutions to the evolutionary variational inequalities is the following (see [19] and [20]):

Theorem 2.2. Let $E$ be a real topological space and $\mathbb{K} \subseteq E$ a convex and nonempty subset. Let $C: \mathbb{K} \rightarrow E^{*}$ be given such that

(1) there exists $A \subseteq \mathbb{K}$ nonempty, compact, and $B \subseteq \mathbb{K}$ compact, convex such that, for every $x \in \mathbb{K} \backslash A$, there exists $y \in B$ with $\langle C(x), y-x\rangle<0$;

and either

(2) $C$ is hemicontinuous in the sense of Ky-Fan; 
or

(3) $C$ is pseudomonotone in the sense of Karamardian and hemicontinuous along line segments.

Then there exists $\bar{x} \in A$ such that $\langle C(\bar{x}), y-\bar{x}\rangle \geq 0$ for all $y \in \mathbb{K}$.

We define the $\left(|L|+\left|L_{1}\right|\right)$-dimensional column vector

$$
v(x(t))=\left(v^{1}(f(\tau)), v^{2}(u(t))\right)
$$

with the $a$-th component, $v_{a}^{1}$, of $v^{1}$ given by

$$
v_{a}^{1}\left(f_{a}(\tau)=\frac{\left.\partial c_{a}\left(f_{a}(\tau)\right)\right)}{\partial f_{a}(\tau)}, \quad \forall a \in L\right.
$$

and the $\alpha$-th component, $v_{\alpha}^{2}$, of $v^{2}$ given by

$$
v_{\alpha}^{2}\left(u_{\alpha}(t)\right)=\frac{\partial \pi_{\alpha}\left(u_{\alpha}(t)\right)}{\partial u_{\alpha}(t)}, \quad \forall \alpha \in L_{1} .
$$
by

If we denote by $\mathscr{L}^{*}$ the dual space of $\mathscr{L}$, on $\mathscr{L}^{*} \times \mathscr{L}$ we define the canonical bi-linear form

$$
\ll G, F \gg:=\int_{0}^{T}\langle G(t), F(t)\rangle d t, \quad G \in \mathscr{L}^{*}, F \in \mathscr{L} .
$$

Adapting Theorem 2.2 to our case, we obtain the following theorem that provides an existence result to evolutionary variational inequality $(2.16)$.

Theorem 2.3. Each of the following conditions is sufficient for the existence of a solution to the evolutionary variational inequality (2.16) (see [22]):

(1) There exist $A \subseteq \mathbb{K}$ compact and $B \subseteq \mathbb{K}$ convex and compact such that $\forall x^{1}(t)=\left(f^{1}(\tau)\right.$, $\left.u^{1}(t)\right) \in \mathbb{K} \backslash A$ there exists

$$
x^{2}(t)=\left(f^{2}(\tau), u^{2}(t)\right) \in B
$$

such that $\ll v\left(x_{1}(t)\right), x_{2}(t)-x_{1}(t) \gg<0 ;$

(2) $v(x(t))$ is pseudomonotone;

(3) $v(x(t))$ is a linear operator on $\mathbb{K}$.

In [23], it was shown that the following uniqueness result holds.

Theorem 2.4. If the function involved in the time-dependent variational inequality (2.16), $v(x(t))$, is strictly pseudo-monotone on $\mathbb{K}$, namely for every pair of distinct points $x^{1}(t), x^{2}(t) \in$ $\mathbb{K}$ we have:

$$
\left\langle v\left(x^{1}(t)\right), x^{2}(t)-x^{1}(t)\right\rangle \geq 0 \Longrightarrow\left\langle v\left(x^{2}(t)\right), x^{2}(t)-x^{1}(t)\right\rangle>0,
$$

then the EVI (2.16) admits a unique solution over the constraint set $\mathbb{K}$.

The following result ensures a sufficient condition to the strictly pseudo-monotonicity of function $v(x(t))$.

Theorem 2.5. If $c_{a}(\cdot)$ and $\pi_{\alpha}(\cdot)$ are strictly convex and differentiable functions for all $a \in L$ and for all $\alpha \in L_{1}$, respectively, then $v(\cdot)$ is a strictly pseudo-monotone function. 
Proof. Let $x^{1}(t), x^{2}(t) \in \mathbb{K}$ be two elements such that $x^{1}(t) \neq x^{2}(t)$ and we suppose that

$$
\begin{aligned}
\left\langle v\left(x^{1}(t)\right), x^{2}(t)-x^{1}(t)\right\rangle \geq 0 \Leftrightarrow & \sum_{a \in L} \frac{\partial c_{a}\left(f_{a}^{1}(\tau)\right)}{\partial f_{a}(\tau)} \times\left[f_{a}^{2}(\tau)-f_{a}^{1}(\tau)\right] \\
& +\sum_{\alpha \in L_{1}} \frac{\partial \pi_{\alpha}\left(u_{\alpha}^{1}(t)\right)}{\partial u_{\alpha}(t)} \times\left[u_{\alpha}^{2}(t)-u_{\alpha}^{1}(t)\right] \geq 0 .
\end{aligned}
$$

We evaluate the following quantity:

$$
\begin{aligned}
\left\langle v\left(x^{2}(t)\right), x^{2}(t)-x^{1}(t)\right\rangle & =\left\langle v\left(x^{2}(t)\right)-v\left(x^{1}(t)\right)+v\left(x^{1}(t)\right), x^{2}(t)-x^{1}(t)\right\rangle \\
& =\left\langle v\left(x^{2}(t)\right)-v\left(x^{1}(t)\right)\right\rangle+\left\langle v\left(x^{1}(t)\right), x^{2}(t)-x^{1}(t)\right\rangle \\
& =\sum_{a \in L}\left(\frac{\partial c_{a}\left(f_{a}^{2}(\tau)\right)}{\partial f_{a}(\tau)}-\frac{\partial c_{a}\left(f_{a}^{1}(\tau)\right)}{\partial f_{a}(\tau)}\right) \times\left[f_{a}^{2}(\tau)-f_{a}^{1}(\tau)\right] \\
& +\sum_{\alpha \in L_{1}}\left(\frac{\partial \pi_{\alpha}\left(u_{\alpha}^{2}(t)\right)}{\partial u_{\alpha}(t)}-\frac{\partial \pi_{\alpha}\left(u_{\alpha}^{1}(t)\right)}{\partial u_{\alpha}(t)}\right) \times\left[u_{\alpha}^{2}(t)-u_{\alpha}^{1}(t)\right] \\
& +\sum_{a \in L} \frac{\partial c_{a}\left(f_{a}^{1}(\tau)\right)}{\partial f_{a}(\tau)} \times\left[f_{a}^{2}(\tau)-f_{a}^{1}(\tau)\right] \\
& +\sum_{\alpha \in L_{1}} \frac{\partial \pi_{\alpha}\left(u_{\alpha}^{1}(t)\right)}{\partial u_{\alpha}(t)} \times\left[u_{\alpha}^{2}(t)-u_{\alpha}^{1}(t)\right] .
\end{aligned}
$$

If $c_{a}(\cdot)$ and $\pi_{\alpha}(\cdot)$ are strictly convex and differentiable functions, then the first two terms of the last expression are strictly greater than zero. Moreover, the sum of the last two terms is greater than or equal to zero for the choice of $x^{1}(t), x^{2}(t) \in \mathbb{K}$. Therefore, quantity (2.18) is strictly grearer than zero. Hence, $v(\cdot)$ is a strictly pseudo-monotone function.

\section{THE ASSOCIATED PROJECTED DYNAMICAL SYSTEM AND THE COMPUTATIONAL PROCEDURE}

In this section, we will describe the relation between the evolutionary variational inequality (2.16) and a particular projected dynamical system. In [23], it was shown that for the case of Hilbert spaces, namely $\mathscr{L}$, the following infinite-dimensional PDS can be associated with the EVI (2.16) as follows:

$$
\frac{d x(t, \tilde{\tau})}{d \tilde{\tau}}=\Pi_{\mathbb{K}}(x(t, \tilde{\tau}),-v(x(t, \tilde{\tau}))), \quad x(t, 0) \in \mathbb{K},
$$

where

$$
\Pi_{\mathbb{K}}(y,-v(y))=\lim _{\delta \rightarrow 0^{+}} \frac{P_{\mathbb{K}}((y-\delta v(y))-y)}{\delta}, \quad \forall y \in \mathbb{K}
$$

with the projection operator $P_{\mathbb{K}}: H \rightarrow \mathbb{K}$ given by

$$
\left\|P_{\mathbb{K}}(z)-z\right\|=\inf _{y \in \mathbb{K}}\|y-z\| .
$$

Following [24], in [25] it was shown that the following theorem holds:

Theorem 3.1. Assume that $K \subseteq H$ is a non-empty, closed and convex subset and $C: \mathbb{K} \rightarrow \mathscr{L}$ is a pseudo-monotone Lipschitz continuous vector field, where $H$ is a Hilbert space. Then the 
solutions of EVI (2.16) are the same as the critical points of the projected differential equation (3.1) that is, they are the functions $x(t) \in \mathbb{K}$ such that

$$
\Pi_{\mathbb{K}}(x(t),-v(t))=0
$$

and vice versa.

According to Theorem 3.1, the solutions to the evolutionary variational inequality: determine $x(t) \in \mathbb{K}$ such that:

$$
\int_{0}^{T}\langle v(x(t)), y(t)-x(t)\rangle \geq 0, \quad \forall y \in \mathbb{K}
$$

are the same as the critical points of the equation:

$$
\frac{d x(t, \tilde{\tau})}{d \tilde{\tau}}=\Pi_{\mathbb{K}}(x(t, \tilde{\tau}),-v(x(t, \tilde{\tau}))),
$$

that are the points such that

$$
\Pi_{\mathbb{K}}(x(t, \tilde{\tau}),-v(x(t, \tilde{\tau}))) \equiv 0 \text { a.e. in }[0, T],
$$

wich are stationary with respect to $\tilde{\tau}$.

In [23], the authors discussed the meaning of the two "times" in (3.3). In particular, they noted that, intuitively, at each instant $t \in[0, T]$, the solution of the evolutionary variational inequality (2.16) represents a static state of the underlying system. As $t$ varies over $[0, T]$, the static states describe one (or more) curves of the equilibria. In contrast, $\tilde{\tau}$ here is the time that describes the dynamics of the system until it reaches one of the equilibria of the curve. In our case, $\tilde{\tau}$ represents the time that the firm takes to adapt its production to excessive demand and, therefore, the delay time in product supplying.

To solve evolutionary variational inequality (2.16), we utilize the approach described in [25], [23], in which the time horizon $T$ is discretized and at each fixed time we then solve the associated static projected dynamical system (see also [26]). For sake of simplicity, we will build the examples with a vector field $v$ satisfying the requirements in Theorem 3.1.

The algorithmic procedure is now described. We select discrete points in time for each example over the interval $[0, T]$. We then apply the Euler method at each discrete time point over the time interval $T$. The Euler method is induced by the general iterative scheme applied in [26] to solve the variational inequality problem. However, this procedure is correct if the continuity of the solution is guaranteed. In the case where the vector field $v(x(t))=A(t) x(t)+B(t)$ is a linear operator, $A(t)$ is a continuous and positive definite matrix in $[0, T]$ and $B(t)$ is a continuous vector, continuity results have been obtained in [27].

In our context, function $v \in L^{2}\left([0, T], \mathbb{R}_{+}^{|L|+\left|L_{1}\right|}\right)$ is a non linear operator. To obtain regularity results, we have to introduce some preliminary definitions.

Definition 3.1. Let $(X, d)$ be a metric space and $Y \subset X$ a closed, nonempty, convex set. A sequence of nonempty, closed, convex sets $Y_{n}$ converges to $Y$ as $n \rightarrow \infty$, i.e. $Y_{n} \rightarrow Y$ in the sense og Kuratowski, if and only if:

(K1) for any $y \in Y$, there exists a sequence $\left\{y_{n}\right\}_{n \in \mathbb{N}}$ converging to $y$ in $X$ such that $y_{n}$ lies in $Y_{n}$ for all $n \in \mathbb{N}$;

(K2) for any subsequece $\left\{y_{n}\right\}_{n \in \mathbb{N}}$ converging to $y$ in $X$, such that $y_{n}$ lies in $Y_{n}$ for all $n \in \mathbb{N}$, then the limit $y$ belongs to $Y$. 
Moreover, we recall that problem (2.16) (see [28]) is also equivalent to the following one: Find $x^{*} \in \mathbb{K}$ such that

$$
\left\langle v\left(x^{*}(t)\right), x(t)-x^{*}(t)\right\rangle \geq 0, \quad \forall x(t) \in \mathbb{K}(t), \text { a.e. in }[0, T],
$$

where

$$
\begin{aligned}
\mathbb{K}(t):=\left\{\begin{array}{l}
x(t) \in \mathbb{R}_{+}^{|L|+\left|L_{1}\right|}: \exists F(\tau) \in \mathbb{R}_{+}^{|P|} \text { and } \\
f_{a}(\tau)=\sum_{p \in P} F_{p}(\tau) \delta_{a p}, \quad \forall a \in L
\end{array}\right. \\
f_{\alpha}(\tau) \leq \bar{u}_{\alpha}(\tau)+u_{\alpha}(t), \quad \forall \alpha \in L_{1}, \\
u_{\alpha}(t) \leq \lambda_{\alpha}(t), \quad \forall \alpha \in L_{1}, \\
f_{\beta}(\tau) \leq \bar{u}_{\beta}(\tau), \quad \forall \beta \in L_{2}, \\
\left.\sum_{p \in P_{w_{k}}} \delta_{a p} F_{p}(\tau) \geq l_{k} I_{k}(t), \quad k=1, \ldots, K\right\} .
\end{aligned}
$$

We suppose that $\mathbb{K}(t)$ satisfies the following assumption:

(K) $\mathbb{K}(t), t \in[0, T]$, is a family of nonempty convex, closed sets of $\mathbb{R}_{+}^{|L|+\left|L_{1}\right|}$ such that $\mathbb{K}\left(t_{n}\right)$ converges to $\mathbb{K}(t)$ in Kuratowski's sense, for each sequence $\left\{t_{n}\right\}_{n \in \mathbb{N}} \subseteq[0, T]$, with $t_{n} \rightarrow$ $t$, as $n \rightarrow \infty$.

Adapting Theorem 4.2 in [29] to our case, we have the following result.

Theorem 3.2. Let $v \in C\left([0, T], \mathbb{R}_{+}^{|L|+\left|L_{1}\right|}\right)$ be a vector-function satisfying the conditions

$$
\|v(x(t))\|_{|L|+\left|L_{1}\right|} \leq A(t)\|x(t)\|_{|L|+\left|L_{1}\right|}+B(t), \quad \text { in }[0, T],
$$

with $B \in C([0, T])$ and $A \in C([0, T])$, and $v$ is a strictly monotone operator. Let $\mathbb{K}(t), t \in[0, T]$, be a family of uniformly bounded sets satisfying condition $(K)$. Then, evolutionary variational inequality (3.4) admits a unique solution $x \in \mathbb{K}$ such that $x \in C\left([0, T], \mathbb{R}_{+}^{|L|+\left|L_{1}\right|}\right)$.

The calculations are performed using the Matlab program. The algorithm has been implemented on a laptop with 1,8 GHz Intel Core i5 dual-core and 8 GB RAM, $1600 \mathrm{MHz}$ DDR3. For the convergence of the method a tolerance of $\varepsilon=10^{-4}$ is fixed. The method has been implemented with the sequence $\left\{\alpha_{\tau}\right\}$ set to $0.1\left\{1, \frac{1}{2}, \frac{1}{2}, \frac{1}{3}, \frac{1}{3}, \frac{1}{3}, \ldots\right\}$.

\section{NUMERICAL EXAMPLES}

In this section we provide two numerical examples, studied in a fixed time interval, using the model described in Section 2.

4.1. Example 1. We suppose to study our model in the time interval $[0,6]$ where the time unit is months and $t=0$ indicates February 2020. The supply chain network is constituted by two manufacturing plants, two distribution centers, two distribution storage centers and three demand markets. The structure of the network is depicted in Figure 2. The links are labeled as 


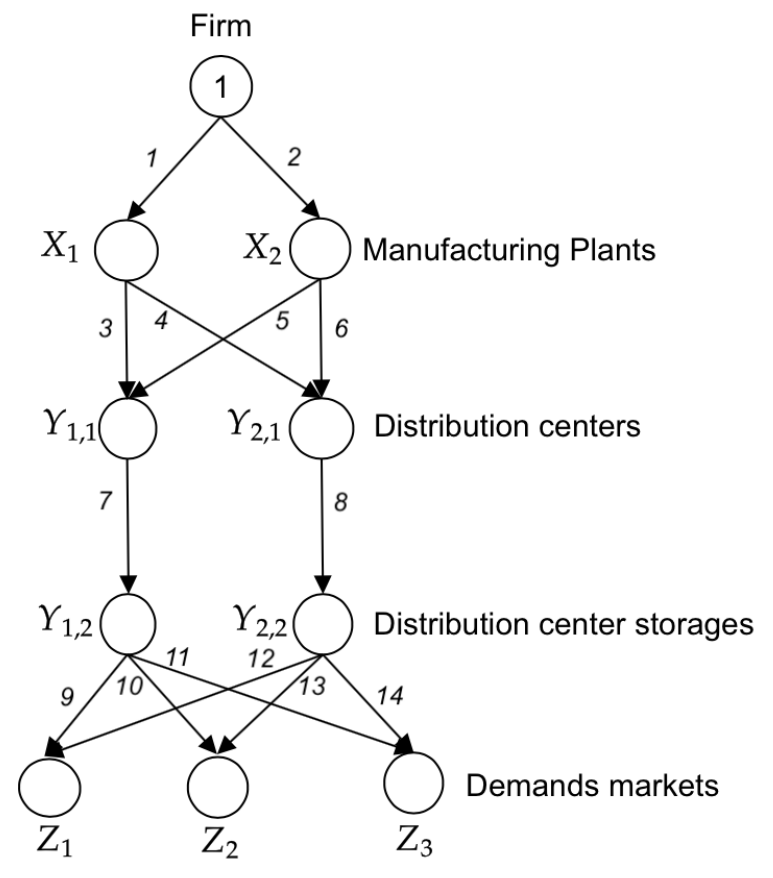

FIgURE 2. Supply Chain Network Topology for the numerical example.

$\{1,2,3, \ldots\}$ and we set $L_{1}=\{1,2,4,5,7,8,10,12,14\}$ and $L_{2}=\{3,6,9,11,13\}$. We have three $\mathrm{O} / \mathrm{D}$ pairs $w_{1}=\left(1, Z_{1}\right), w_{2}=\left(1, Z_{2}\right)$ and $w_{3}=\left(1, Z_{3}\right)$. Hence, we have:

$$
\begin{gathered}
P_{w_{1}}=\left\{p_{1}=\{1,3,7,9\}, p_{2}=\{1,4,8,12\}, p_{3}=\{2,5,7,9\}, p_{4}=\{2,6,8,12\}\right\} ; \\
P_{w_{2}}=\left\{p_{5}=\{1,3,7,10\}, p_{6}=\{1,4,8,13\}, p_{7}=\{2,5,7,10\}, p_{8}=\{2,6,8,13\}\right\} ; \\
P_{w_{3}}=\left\{p_{9}=\{1,3,7,11\}, p_{10}=\{1,4,8,14\}, p_{11}=\{2,5,7,11\}, p_{12}=\{2,6,8,14\}\right\} .
\end{gathered}
$$

We set our scenario in Italy. The territory of Italy is divided into 20 regions; during the Covid-19 pandemic, the most affected regions of Italy were the northern ones, with the highest number of infected people ever recorded in Lombardy (see [30]) We suppose that the demand markets are the individual regions. We consider three demand markets that differ in the degree of spread of the virus. Specifically, we choose the three regions Molise, Sicily and Lombardia. The first region represents the demand market in which a small number of infections has been recorded; the second region represents the demand market where there was a sustained number of infections and the third region represents the demand market where the higher number of infections has been recorded.

As already announced in Section 2, the request in the demand markets is proportional to the number of the trend of infections in the same demand market, that we indicate with $I_{k}(t)$, for all $k=1, \ldots, K$ and $t \in[0,6]$. To obtain a functional expression of $I_{k}(t)$, we utilize a quadratic interpolation where data are the number of infections at instants $t_{1}=0, t_{2}=3$ and $t_{3}=6$, i.e. February, May and August. According to [31], we obtain the following table: 


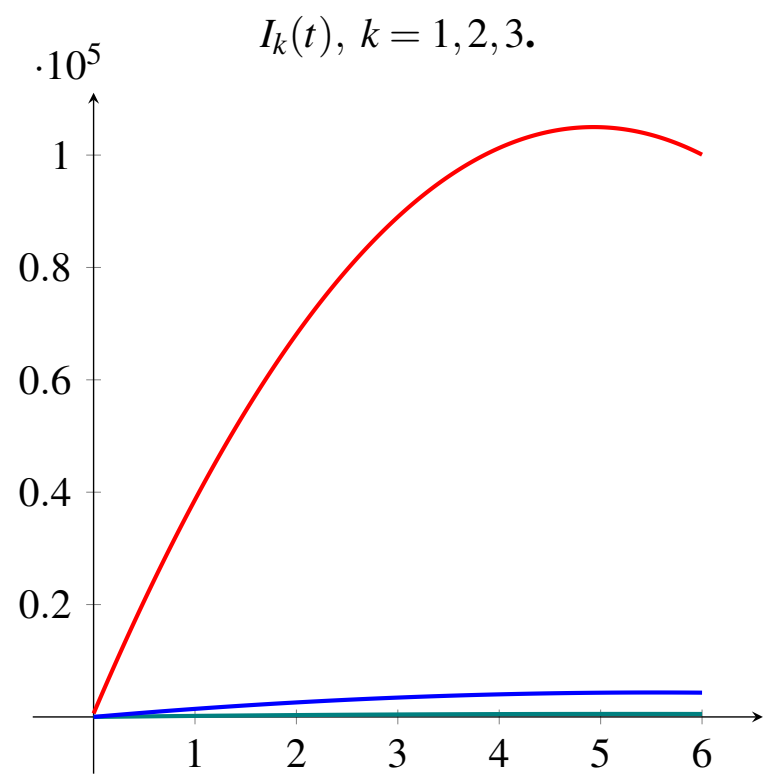

FIGURE 3. Graphic representation of $I_{1}(t)$ (in green), $I_{2}(t)$ (in blue) and $I_{3}(t)$ (in red).

\begin{tabular}{cccc}
\hline $\begin{array}{l}\text { Region } \\
\text { Demand Markets }\end{array}$ & $\begin{array}{l}\text { Number of } \\
\text { infections at } t_{1}\end{array}$ & $\begin{array}{l}\text { Number of } \\
\text { infections at } t_{2}\end{array}$ & $\begin{array}{l}\text { Number of } \\
\text { infections at } t_{3}\end{array}$ \\
\hline Molise & 0 & 436 & 525 \\
\hline Sicily & 4 & 3,443 & 4,317 \\
\hline Lombardia & 615 & 88,968 & 100,075
\end{tabular}

TABLE 1. Number of infections in the demand markets in the three chosen times (data obtained by [31]).

Using data in Table 1, we obtain the following expression for $I_{k}(t), k=1,2,3$ :

$$
\begin{gathered}
I_{1}(t)=203.167 t-19.2778 t^{2} ; \\
I_{2}(t)=4+1,573.83 t-142.5 t^{2} \\
I_{3}(t)=615+42,337.3 t-4,293.44 t^{2} .
\end{gathered}
$$

Figure 3 shows their graphic representation. Moreover, we choose $l_{1}=2, l_{2}=4$ and $l_{3}=9$. Now let us introduce the firm reaction time function:

$$
w(t)=\sum_{k=1}^{3} \frac{d I_{k}(t)}{d t}=44,114.3-4,455.21 t,
$$

that is, the firm reaction time is the sum of the rate of change of infections in all demand markets.

Moreover, we introduce the delay function

$$
h(t, w(t))=5 t+0.0001 w(t)=4.41+0.054 t .
$$

Then,

$$
\tau=4.41+1.05 t
$$



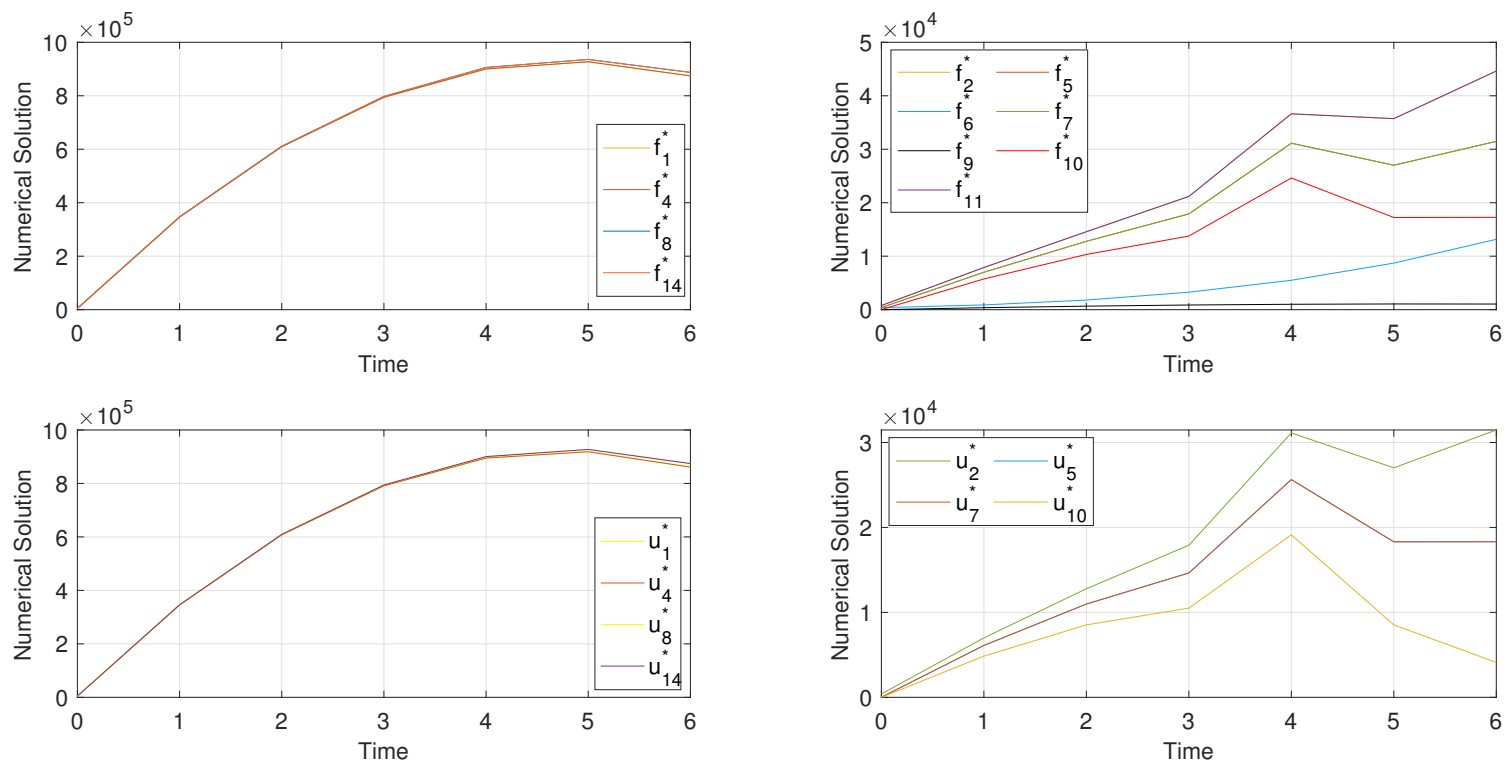

FIGURE 4. Numerical solutions for Example 1.

We assume the following cost functions are given:

$$
c_{a}\left(f_{a}(\tau)\right)=0.5 f_{a}^{2}(\tau)+f_{a}(\tau), \quad \forall a \in L .
$$

Moreover, we assume that the following investment capacity functions are given:

$$
\pi_{\alpha}\left(u_{\alpha}(t)\right)=0.5 u_{\alpha}^{2}(t)+u_{\alpha}(t), \quad \forall \alpha \in L_{1} .
$$

The existing capacities are $\bar{u}_{a}(\tau)=\zeta^{4}(t), \forall a \in L$ and, finally, the upper bounds for additional capacity are $\lambda_{\alpha}(t)=9.4 \times 10^{5}, \forall t \in[0,6], \forall \alpha \in L_{1}$. The optimal solution is shown in Figure 4.

We observe that Figure 4 shows, at every instant $t \in[0,6]$, the retarded optimal flows $f_{a}(4.41+$ $1.05 t), a \in L$, and the added capacity on arcs. This means that, at instant $\mathrm{t}=1$, for example, the request in the demand markets is satisfied at the instant $t=4.41+1.05=5.46$, etc.

As shown in Figure 4, the huge increase in request in the demand markets forces the company to invest in the arcs of the set $L_{1}$ to increase their capacity, except for the $\operatorname{arc} a=12$, which serves the demand market $k=1$ ( $\operatorname{arc} a=12$ is not reported in Figure 4 because it is null over time). This is due to the fact that the request in this demand market is relatively low and, therefore, the company manages to merge the requested product only in the arc $a=9$, for which, instead, the additional capacity is not provided. Indeed, the flow on arc $a=9$ increases from $f_{9}^{*}(\zeta(0))=0$ to $f_{9}^{*}(\zeta(6))=1052$, that represents the total demand in demand market 1 at time $t=6$.

Moreover, the optimal flows on arcs with non-increasing capacity $a=3$ and $a=13$ are null over time and this means that, in terms of costs, the company prefers to invest in arcs for which it is possible to increase the capacity and use the paths that include these arcs. This preference can also be deduced from the Table 2, which shows the flows on the paths at each instant $t \in[0,6]$. 


\begin{tabular}{cccccccc}
\hline & $t=0$ & $t=1$ & $t=2$ & $t=3$ & $t=4$ & $t=5$ & $t=6$ \\
\hline$F_{1}^{*}$ & 0 & 0 & 0 & 0 & 0 & 0 & 0 \\
$F_{2}^{*}$ & 0 & 0 & 0 & 0 & 0 & 0 & 0 \\
$F_{3}^{*}$ & 0 & 367.80 & 658.44 & 872.00 & 1008 & 1067.80 & 1052 \\
$F_{4}^{*}$ & 0 & 0 & 0 & 0 & 0 & 0 & 0 \\
$F_{5}^{*}$ & 0 & 0 & 0 & 0 & 0 & 0 & 0 \\
$F_{6}^{*}$ & 0 & 0 & 0 & 0 & 0 & 0 & 0 \\
$F_{7}^{*}$ & 16 & 5740.00 & 10324 & 13768 & 24624 & 17240 & 17264 \\
$F_{8}^{*}$ & 0 & 0 & 0 & 0 & 0 & 0 & 0 \\
$F_{9}^{*}$ & 0 & 0 & 0 & 0 & 0 & 0 & 0 \\
$F_{10}^{*}$ & 4778.54 & 346144.53 & 609442.85 & 794331.93 & 900429.86 & 927269.39 & 874351.93 \\
$F_{11}^{*}$ & 378.22 & 888.73 & 1796.07 & 3266.53 & 5495.56 & 8707.80 & 13157.03 \\
$F_{12}^{*}$ & 378.22 & 888.73 & 1796.07 & 3266.53 & 5495.56 & 8707.80 & 13157.03 \\
\hline
\end{tabular}

TABLE 2. Optimal path flows over time for Example 1.

4.2. Statistical Analysis. In this subsection, we conduct a statistical analysis on arc flows, considering different expressions for arc cost functions and for investment capacity functions. To do this, we consider the general quadratic expression of these costs:

$$
\begin{gathered}
c_{a}\left(f_{a}(t)\right)=\gamma_{a}\left(f_{a}(t)\right)^{2}+\phi_{a} f_{a}(t)+d_{a}, \quad \forall a \in L ; \\
\pi_{\alpha}\left(u_{\alpha}(t)\right)=\kappa_{\alpha}\left(u_{\alpha}(t)\right)^{2}+\varphi_{\alpha} u_{\alpha}(t)+q_{\alpha}, \quad \forall \alpha \in L_{1} .
\end{gathered}
$$

We set:

$$
d_{a}=0, \quad q_{\alpha}=0, \quad \forall a \in L, \forall \alpha \in L_{1},
$$

and we vary the coefficients $\gamma_{a}, \kappa_{\alpha}$, considering a random uniform distribution in the interval [1;50], and $\phi_{a}$ and $\varphi_{\alpha}$, considering a random uniform distribution in the interval [1;30]. We have solved 15 different instances and we have obtained the optimal solutions as in Figure 5 and Figure 6.

Figure 5 and Figure 6 represent the optimal flows on $\operatorname{arcs} a=1, \ldots, 6$ and $a=7, \ldots, 14$, respectively.

We are interested on the calculation of expectation and variance of arc flows. As we can see from Figure 5, arc flows $f_{a}, a=7, \ldots, 14$, are relatively stable and are subject to small variations only between the instants $t=5$ and $t=6$. The aforesaid arcs represent the final arcs of the supply chain network in Figure 2. Although the costs vary randomly, the company, forced to satisfy the requests in the demand markets, does not have any possibility to significantly vary the flows on these arcs, due to their limited capacity.

Arcs $a=1$ and $a=2$ are complementary: the firm, based on the costs on these arcs, decides whether to merge a greater flow to the first or to the second one, in line with its objective of minimizing total costs. For these costs we obtain the expectactions and variances of optimal arc flows $f_{1}^{*}$ and $f_{2}^{*}$ in Table 3 , calculated at every istant $t=1, \ldots, 6$. 

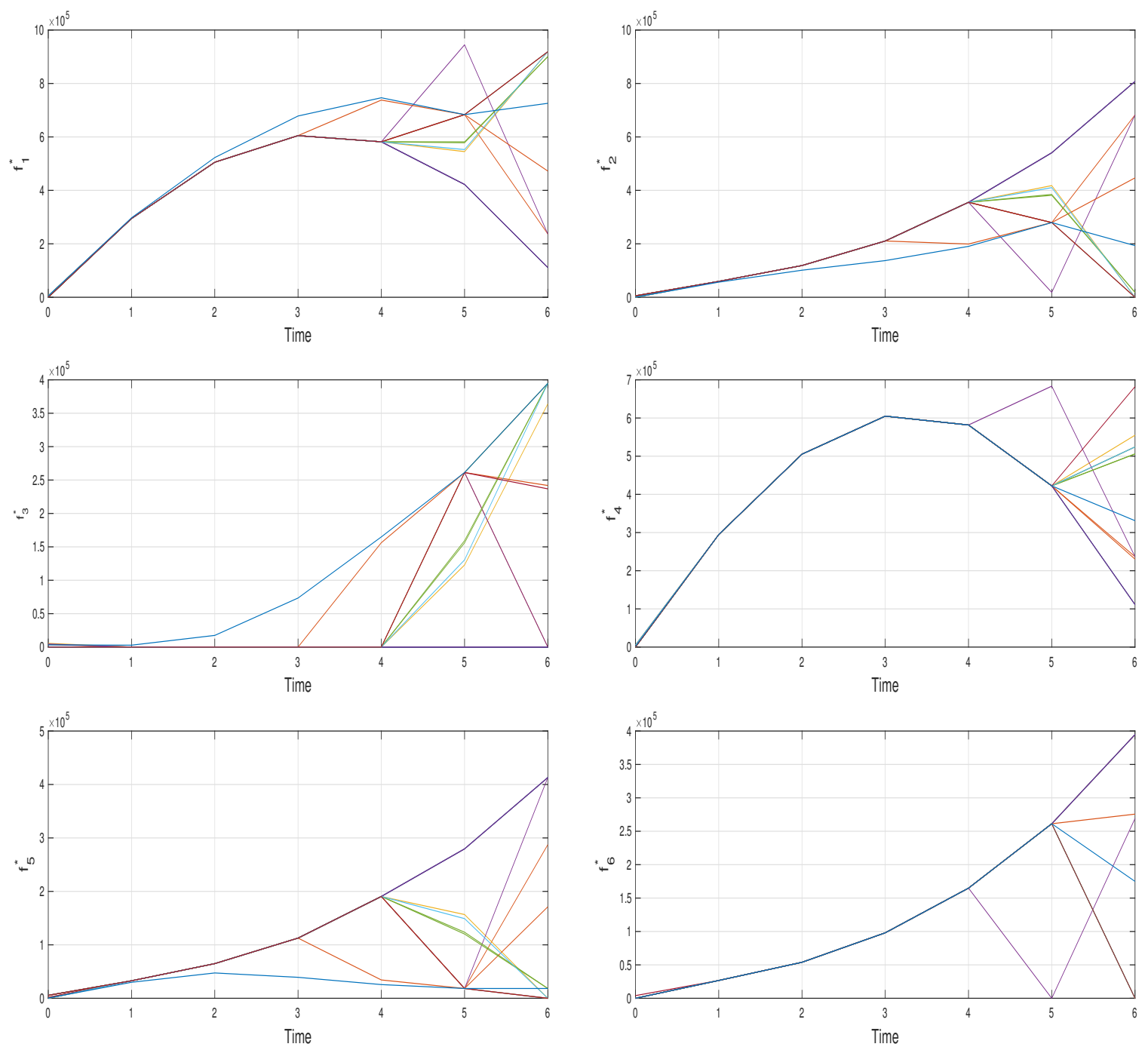

FIGURE 5. Random instances for arcs costs functions $c_{a}, a \in\{1, \ldots, 6\}$.

\begin{tabular}{lllll}
\hline & $\bar{f}_{1}^{*}$ & $\bar{f}_{2}^{*}$ & $\operatorname{Var}\left(f_{1}^{*}\right)$ & $\operatorname{Var}\left(f_{2}^{*}\right)$ \\
\hline$t=0$ & $2.4 \times 10^{3}$ & $3.17 \times 10^{3}$ & $7.15 \times 10^{6}$ & $7.15 \times 10^{6}$ \\
$t=1$ & $2.95 \times 10^{5}$ & $5.92 \times 10^{4}$ & $5.36 \times 10^{5}$ & $5.36 \times 10^{5}$ \\
$t=2$ & $5.11 \times 10^{5}$ & $1.17 \times 10^{5}$ & $2.03 \times 10^{7}$ & $2.03 \times 10^{7}$ \\
$t=3$ & $5.97 \times 10^{5}$ & $2.06 \times 10^{5}$ & $3.59 \times 10^{8}$ & $3.59 \times 10^{8}$ \\
$t=4$ & $6.03 \times 10^{5}$ & $3.33 \times 10^{5}$ & $3.19 \times 10^{9}$ & $3.19 \times 10^{9}$ \\
$t=5$ & $5.99 \times 10^{5}$ & $3.63 \times 10^{5}$ & $2.09 \times 10^{10}$ & $2.09 \times 10^{10}$ \\
$t=6$ & $5.67 \times 10^{5}$ & $3.5 \times 10^{5}$ & $1.37 \times 10^{11}$ & $1.37 \times 10^{11}$
\end{tabular}

TABLE 3. Expectactions and Variances for optimal arc flows $f_{1}^{*}$ and $f_{2}^{*}$. 

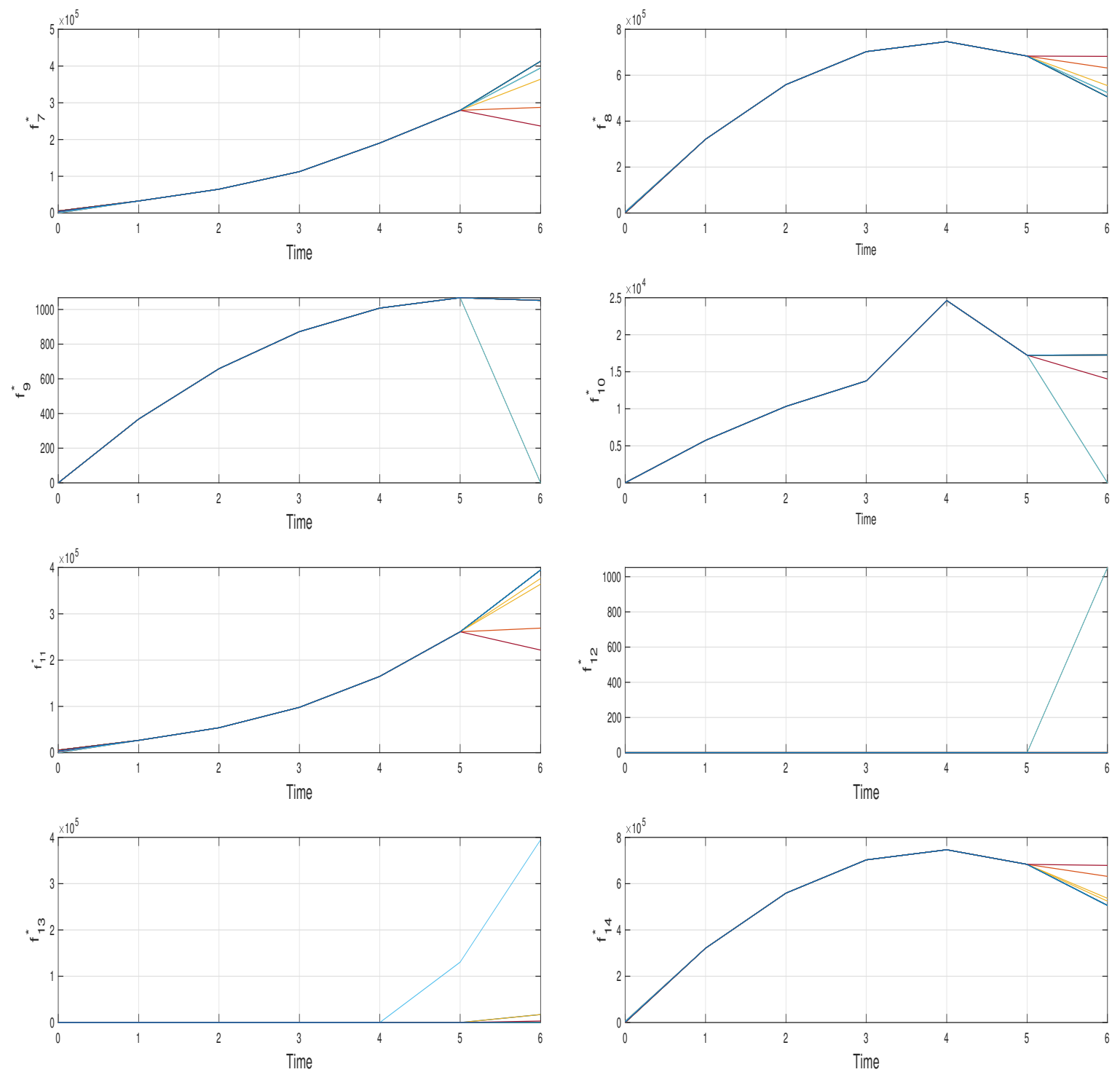

FIGURE 6. Random instances for arcs costs functions $c_{a}, a \in\{7, \ldots, 14\}$.

According to Table 3, the firm decides to merge more product flow on the arc $a=1$, as the average value of the flows on such an arc is much greater than the one on the $\operatorname{arc} a=2$. Further, the variance of the flows on these arcs is very high. Indeed, in some instances, the company decides to merge the entire flow on a single arc, keeping null the flow on the complementary arc and vice versa. Similar considerations can be drawn for arcs $a=3,4,5,6$.

This result suggests to us that the optimal solution for each instance is strictly affected by the cost function variations and this is consistent with the mathematical model presented in Section 2. 


\begin{tabular}{cccccccc}
\hline & $t=0$ & $t=1$ & $t=2$ & $t=3$ & $t=4$ & $t=5$ & $t=6$ \\
\hline$f_{3}^{*}$ & 1387.94 & 88507.71 & 156004.62 & 203876.51 & 234263.51 & 240748.44 & 229745.74 \\
$f_{4}^{*}$ & 1387.71 & 88507.35 & 156004.26 & 203876.15 & 234263.15 & 240748.12 & 229745.42 \\
$f_{5}^{*}$ & 1387.78 & 88507.54 & 156004.45 & 203876.34 & 234263.34 & 240748.27 & 229745.57 \\
$f_{6}^{*}$ & 1387.55 & 88507.18 & 156004.09 & 203875.98 & 234262.98 & 240747.95 & 229745.25 \\
\hline
\end{tabular}

TABLE 4. Optimal arc flows over time for Example 2.

4.3. Sentivity Analysis. We finally conduct a sensitivity analysis on arc flows. First, we suppose to consider the same data as in Example 1, except that we now suppose that the firm can increase the capacity on each arc, that is $L_{1}=L$. We introduce the following investment capacity functions for all arcs:

$$
\pi_{a}\left(u_{a}(t)\right)=0.5 u_{a}^{2}(t)+u_{a}(t), \quad \forall a \in L .
$$

In particular, we focus on arcs 3,4,5 and 6, that represent the central arcs of the network in Figure 2. Their optimal values read as in Table 4.

As it is possible to see, the company, faced with a huge and sudden increase in demand, having the possibility of increasing the capacity on each arc that forms the supply network, decides indiscriminately to use all the arcs in the same way.

For arcs $a=3$ and $a=5$ we vary the coefficients of the quadratic terms, uniformly in the interval $[1,10]$ with increments of 0.5 units.

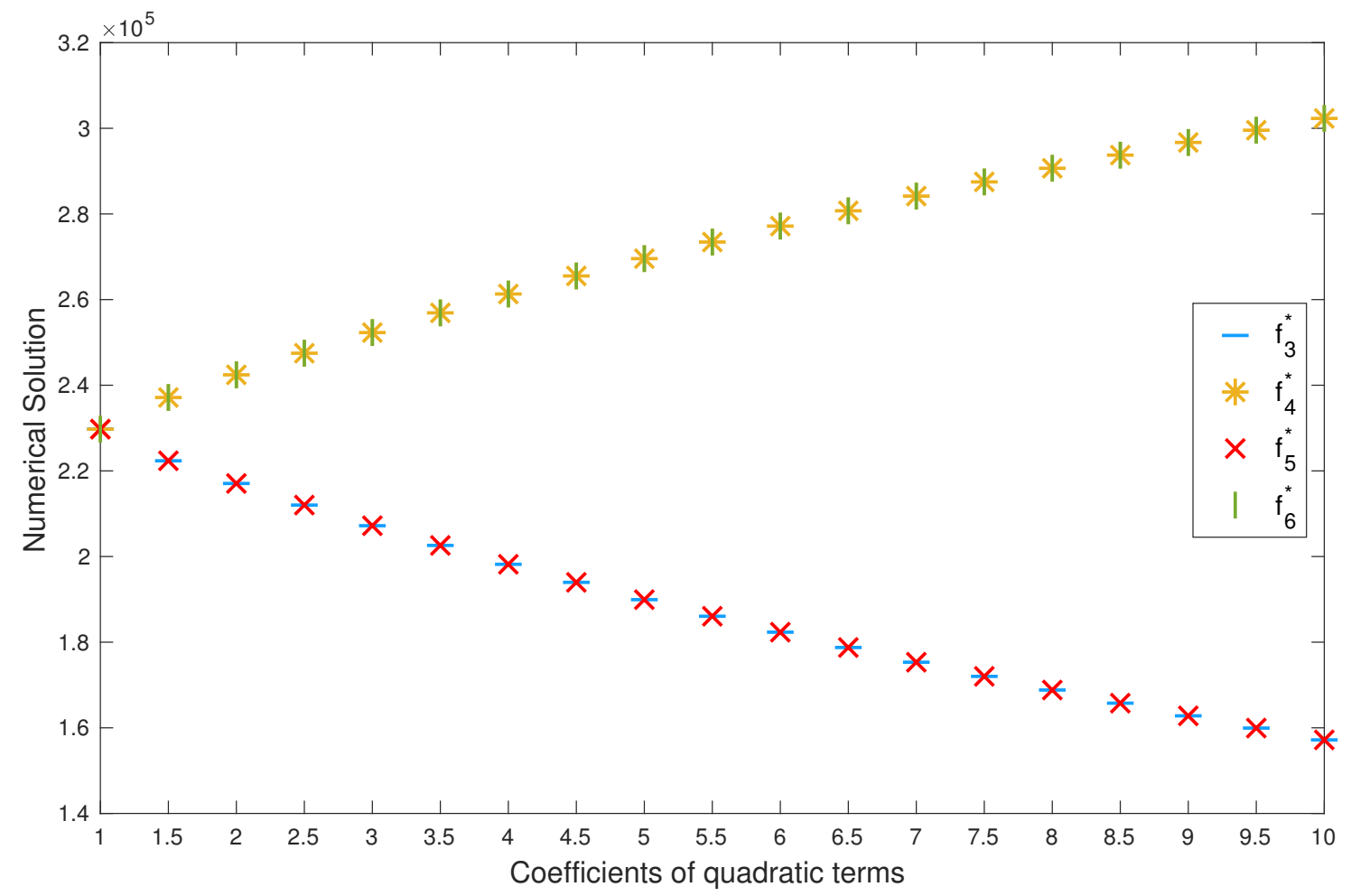

FIGURE 7. Sensitivity analysis for coefficients of quadratic terms of $c_{3}$ and $c_{5}$ variations. 
The values for the optimal flows on $\operatorname{arcs} a=3$ and $a=5$ for time $t=6$ are reported in Figure 7. Figure 7 shows that, as the coefficient of quadratic terms for arc cost functions $c_{3}$ and $c_{5}$ increase, the optimal flows on these arcs decrease uniformly, while, at the same time, the optimal flows on $\operatorname{arcs} a=4$ and $a=5$ increase. This result suggests to us that the firm prefers not to invest in the arcs $a=3$ and $a=5$, but decides to merge the product flows into the arcs with lower costs. This is consistent with the objective of the mathematical model proposed in Section 2.

\section{CONCLUSION}

In this paper, we developed an optimization model consisting in a dynamic supply chain network for PPE studied in a time interval $[0, T]$, in which arc flows, path flows and existing capacity on arcs depend both on time and on a delay function, in order to find the optimal flows and the optimal adding capacities on arcs that firm has to adopt to satisfy the huge and immediate increase in request for such devices in demand markets due to the spread of the COVID-19 disease. We obtained a minimization problem and the related "retarded" evolutionary variational inequality. Furthermore, we introduced the associated infinite-dimensional projected dynamical system to determine a computational procedure to find the optimal variables of the model. A concrete example, set in Italy, was presented and solved and both statistical and sensitivity analysis on arc flows were conducted. The optimal solution showed the effectiveness of the model and the need for the firm to improve its supply and production capacities.

In a future research it would be interesting to introduce the Lagrange multipliers associated with the constraint and make an analysis of their values, so to examine further features of the model.

\section{Acknowledgments}

The authors appreciate very much the feedback and comments received by the referees on an earlier version of this paper. The research was partially supported by the research project "Programma ricerca di ateneo UNICT 2020-22 linea 2 (OMNIA)" University of Catania. This support was gratefully acknowledged.

\section{REFERENCES}

[1] C.T. Lopez, Domestic N95 mask production expected to exceed 1 billion in 2021, 2020. Available at: https://www.defense.gov/Explore/News/Article/Article/2215532/ domestic-n95-mask-production-expected-to-exceed-1-billion-in-2021/

[2] A. Nagurney, P. Daniele, E.A. Flores, V. Caruso, A variational equilibrium network framework for humanitarian organizations in disaster relief: Effective product delivery under competition for financial funds, In: Dynamics of Disasters: Algorithmic Approaches and Applications, Kotsireas, I.S., Nagurney, A., Pardalos, P.M., (ed.), pp. 109-133, Springer, Switzerland, 2018.

[3] A. Nagurney, M. Salarpour, J. Dong, P. Dutta, Competition for medical supplies under stochastic demand in the Covid-19 pandemic, In: Nonlinear Analysis and Global Optimization, Rassias, T.M., Pardalos, P.M., (ed.), Springer, Switzerland, 2021.

[4] A. Nagurney, M. Salarpour, J. Dong, L.S. Nagurney, A stochastic disaster relief game theory network model, Oper. Res. Forum 1 (2020), 1-33.

[5] A. Nagurney, M. Yu, D. Besik, Supply chain network capacity competition with outsourcing: A variational equilibrium framework, J. Global Optim. 69 (2017), 231-254. 
[6] A. Nagurney, M. Yu, Q. Qiang, Supply chain network design for critical needs with outsourcing, Papers in Regional Science, 90 (2010), 123-142.

[7] A. Nagurney, L.S. Nagurney, A mean-variance disaster relief supply chain network model for risk reduction with stochastic link costs, time targets, and demand uncertainty, In: Dynamics of Disasters: Key Concepts, Models, Algorithms, and Insights, I.S. Kotsireas, A. Nagurney, P.M. Pardalos, (ed.), pp. 231-255, Springer, Switzerland, 2016.

[8] A. Nagurney, A.H. Masoumi, M. Yu, An integrated disaster relief supply chain network model with time targets and demand uncertainty, In: Regional Science Matters: Studies Dedicated to Walter Isard, P. Nijkamp, A. Rose, K. Kourtit, (ed.), pp. 287-318, Springer, Switzerland, 2015.

[9] F. He, J. Zhuang, Balancing pre-disaster preparedness and post-disaster relief, Eru. J. Oper. Res. 252 (2016), 246-256.

[10] A. Nagurney, M. Salarpour, A multicountry, multicommodity stochastic game theory network model of competition for medical supplies inspired by the Covid-19 pandemic, Int. J. Production Economics, in press.

[11] P. Daniele, Dynamic Networks and Evolutionary Variational Inequalities, Edward Elgar Publishing, Cheltenham, 2006.

[12] T.L. Friesz, D. Bernstein, T.E. Smith, R.L. Tobin, B.W. Wie, A variational inequalities formulation of the dynamic network user equilibrium problem, Oper. Res. 41 (1993), 179-191.

[13] F. Raciti, Equilibrium in Time-Dependent Traffic Networks with Delay in Equilibrium Problems: Nonsmooth Optimization and Variational Inequality Models, F. Giannesi, A. Maugeri and P.M. Pardalos (ed.), pp. 247253, Kluwer Academic Publishers, The Netherlands, 2001.

[14] L. Scrimali, Quasi-variational inequalities in trasportation networks, Math. Models Methods Appl. Sci. 14 (2004), 1541-1560.

[15] L. Scrimali, Quasi-Variational Inequalities Applied to Retarded Equilibria in Time-Dependent Traffic Problems, in Variational Analysis and Applications, F. Giannesi and A. Maugeri (ed.), pp. 1007-1023, Springer, New York, 2005.

[16] P. Daniele, Evolutionary Variational Inequalities and Applications to complex dynamic multi-level models, Trans. Res. Part E, 46 (2010), 855-880.

[17] A. Barbagallo, P. Daniele, A. Maugeri, Variational formulation for a general dynamic financial equilibrium problem: Balance law and liability formula, Nonlinear Anal. 75 (2012), 1104-1123.

[18] P. Daniele, Evolutionary variational inequalities and economic models for demands-supply markets, Math. Models Methods Appl. Sci. 13 (2003), 471-489.

[19] P. Daniele, A. Maugeri, W. Oettli, Variational inequalities and time-dependent traffic equilibria, C. R. Acad. Sci. Paris. 326 (1998), 1059-1062.

[20] P. Daniele, A. Maugeri, W. Oettli, Time-dependent variational inequalities, J. Optim. Theory Appl. 104 (1999), 543-555.

[21] A. Maugeri, F. Raciti, On existence theorems for monotone and nonmonotone variational inequalities, J. Convex Anal. 16 (2009), 899-911.

[22] W. Oettli, D. Schläger, Generalized Vectorial Equilibria and Generalized Monotonicity, in Functional Analysis with Current Applications, M, Brokate and A.H. Siddiqi (ed.), pp. 145-154, Longman, Harlow, 1998.

[23] M.-G. Cojocaru, P. Daniele, A. Nagurney, Double-layered dynamics: A unified theory of projected dynamical systems and evolutionary variational inequalities, Eur. J. Oper. Res. 175 (2006), 494-507.

[24] M.-G. Cojocaru, L.B. Jonker, Existence of solutions to projected differential equations in Hilbert spaces, Proc. Amer. Math. Soc. 132 (2004), 183-193.

[25] M.-G. Cojocaru, P. Daniele, A. Nagurney, Projected dynamical systems, evolutionary variational inequalities, applications, and a computational procedure, In Chinchuluun A., Pardalos P.M., Migdalas A., Pitsoulis L. (ed.) Pareto Optimality, Game Theory And Equilibria. Springer Optimization and Its Applications, vol. 17, pp. 387-406, Springer, New York, 2005.

[26] A. Nagurney, D. Zhang, Projected Dynamical Systems and Variational Inequalities with Applications, Kluwer Academic Publishers, Boston, 1996.

[27] A. Barbagallo, Regularity Results for Time-dependent Variational and Quasivariational Inequalities and Computational Procedures, Department of Mathematics and Computer Sciences, University of Catania, Sicily, 2005. 
[28] A. Maugeri, C. Vitanza, Time-dependet equilibrium problems, In: A. Chinchuluun, P. Pardalos, A. Migdalas, L. Pitsoulis (Ed.), Pareto Optimality Game Theory and Equilibria, in: Springer Optimization and its Applications Series, pp. 249-266, Springer, 2008.

[29] A. Barbagallo, On the regularity of retarded equilibria in time dependent traffic equilibrium problems, Nonlinear Anal. 71 (2009), 2406-2417.

[30] Ministero della Salute, Nuovo coronavirus. http://www.salute.gov.it/portale/ nuovocoronavirus/dettaglioContenutiNuovoCoronavirus . jsp?area=nuovoCoronavirus\& id=5351\&lingua=italiano\&menu=vuoto

[31] Ministero della Salute, https://www.epicentro.iss.it/coronavirus/sars-cov-2-dashboard 